\title{
Responses of Heterodera glycines and Meloidogyne incognita Infective Juveniles to Root Tissues, Root Exudates, and Root Extracts from Three Plant Species
}

Congli Wang, Key Laboratory of Mollisols Agroecology, Northeast Institute of Geography and Agroecology, Chinese Academy of Sciences, Harbin 150081, China; and, Edward P. Masler ${ }^{\dagger}$ and Stephen T. Rogers, United States Department of Agriculture, Agricultural Research Service, Mycology and Nematology Genetic Diversity and Biology Laboratory, Beltsville, MD, 20705

\begin{abstract}
The infective juvenile (J2) stage of endoparasitic plant nematodes uses plant chemical signals, released from roots, to localize and infect hosts. We examined the behaviors of soybean cyst nematode (Heterodera glycines) and root-knot nematode (Meloidogyne incognita) $\mathrm{J} 2$ in the presence of root signals from marigold (Tagetes patula), soybean (Glycine max), and pepper (Capsicum annuum). Signals were obtained from sources commonly used in phytoparasitic nematode chemotaxis studies: root tips, root exudates, and root extracts. Root tips from each plant species attracted $M$. incognita but $H$. glycines was attracted only to soybean. In contrast, root exudates prepared from marigold, pepper, or soybean

seedlings were attractive to $H$. glycines but were repellent to $M$. incognita. Root extracts had the same effect as exudates. Fractionation of exudates by reversed-phase high-performance liquid chromatography (HPLC) (acetonitrile $\left[\mathrm{CH}_{3} \mathrm{CN}\right]$ and $0.1 \%$ trifluoroacetic acid) revealed highly polar and less polar components affecting behaviors. Fractions eluting at $12 \% \mathrm{CH}_{3} \mathrm{CN}$ from all three plants attracted $H$. glycines and repelled $M$. incognita. None of the less polar HPLC fractions $(>15 \%$ $\mathrm{CH}_{3} \mathrm{CN}$ ) affected $H$. glycines but those from $G$. max and T. patula repelled $M$. incognita. Differences among exudates and effects of fractionation on behavior are discussed.
\end{abstract}

The infective juvenile (J2) stage of soil-dwelling plant-parasitic nematodes must negotiate the complex environment of the rhizosphere, where chemical and other signals influence multitrophic behaviors among soil microorganisms and plants (Bais et al. 2006; Rasmann et al. 2012). Chemical cues serve as primary signals for nematode guidance, and prominent among these are plant semiochemicals influencing nematode behaviors important to survival, including host location and infection (Jones 2002; Perry 1996, 1997; Perry and Curtis 2013). The J2 stages of root-penetrating endoparasites such as the soybean cyst nematode Heterodera glycines and root knot nematode Meloidogyne incognita are highly vulnerable to the risk of starvation and other environmental stresses in the time between hatching and plant entry. Consequently, efficient chemotaxis for location of host plants is crucial. Cyst and root knot nematodes make up the most economically damaging groups of plant-parasitic nematodes (Jones et al. 2013), and understanding their responses to plant root signals is an important component in discovering novel controls. With global losses from plant nematode damage persistently approaching the $\$ 100$ billion mark annually (Nicol et al. 2011), and with restrictions placed upon the use of toxic nematicides, the exploration of alternative management strategies, including effects of the massive array of phytochemicals as well as details of nematode chemotaxis (Chitwood 2002; Grundler et al. 1991; Ntalli and Caboni 2012; Reynolds et al. 2011; Wuyts et al. 2006; Yang et al. 2016), has increased urgency.

${ }^{\dagger}$ Corresponding author: E. P. Masler; E-mail: edward.masler@ars.usda.gov

Funding: This research was partially supported by United States Department of Agriculture, Agricultural Research Service. Partial support also was provided by the National Science Foundation of China (grant 31471749) and the One Hundred Talent Grant Program, Chinese Academy of Sciences to C. Wang.

Mention of trade names or commercial products in this publication is solely for the purpose of providing specific information and does not imply recommendation or endorsement by the U.S. Department of Agriculture.

Accepted for publication 5 April 2018.

This article is in the public domain and not copyrightable. It may be freely reprinted with customary crediting of the source. The American Phytopathological Society, 2018.
Examination of the complex behaviors associated with plant signal detection and nematode chemotaxis through experiments designed to simplify interactions and quantify specific behaviors has been productive (Dalzell et al. 2011; Farnier et al. 2012; Masler et al. 2017; Wang et al. 2010; Winter et al. 2002). These approaches frequently involve plant roots or root preparations to provide chemical signals in vitro. We were interested in comparing the effects of various root preparation types and plant sources on the behaviors of $H$. glycines and $M$. incognita $\mathrm{J} 2$. This is the first report of direct comparisons of nematode responses to three sources of root chemicals-root tips, root extracts, and root exudates-from multiple plant species. Evidence is presented for significant differences in $H$. glycines and $M$. incognita response behaviors, and in the chemical nature of plant materials assayed.

\section{Materials and Methods}

Nematode cultures. Plants were grown in a greenhouse in sandfilled beakers using the constant moisture system of Sardanelli and Kenworthy (1997) at $26^{\circ} \mathrm{C}$ and a photoperiod of 16 to $14 \mathrm{~h}$ of light and 8 to $10 \mathrm{~h}$ of darkness. The southern root-knot nematode $M$. incognita (Salisbury, Maryland, race 1) was reared on pepper (Capsicum annuum 'PA 136') and the soybean cyst nematode (H. glycines, NL1-RHp) was reared on soybean (Glycine max 'Kent'). Inoculation rate was approximately 5,000 eggs plant ${ }^{-1}$, with soybean harvested at 5 to 7 weeks and pepper at 6 to 8 weeks after inoculation. Cysts were collected by gently rubbing roots under a stream of tap water; then, cysts were rinsed thoroughly in autoclaved tap water (ATW) and crushed to release eggs. Eggs were then collected on a 500-mesh sieve, rinsed with ATW, and transferred to hatching chambers. $M$. incognita eggs were collected by picking egg masses from roots using a jeweler's forceps, then releasing the eggs by gentle shaking in tap water. Eggs were rinsed with ATW and transferred to hatching chambers comprising 6-well assay plates (Falcon B-D, Franklin Lakes, NJ) with each well-fitted with a sieve. Sieves were prepared using Netwell plate (Corning Costar; Corning Inc., Corning, NY) circular inserts, which have bottom surfaces of $500-\mu \mathrm{m}$ pore polyester mesh. A circle of $30-\mu \mathrm{m}$ pore nylon mesh (Spectrum Laboratories, Rancho Dominguez, CA) was secured on top of the $500-\mu \mathrm{m}$ mesh with a polypropylene ring, and the modified sieves were suspended in plate wells containing $2 \mathrm{ml}$ of ATW. Eggs (approximately 100,000 sieve ${ }^{-1}$ ) were added by pipette to the surface of the nylon mesh. Newly hatched $\mathrm{J} 2$ passed through the nylon mesh into the plate well and were collected daily. $\mathrm{J} 2$ were used in bioassays on the day of collection. 
Plant rearing. Plants used as phytochemical sources included the hosts $C$. annuum and G. max, plus marigold (Tagetes patula 'Golden Guardian'; Park Seed, Greenwood, SC) for its nematicidal and biological control properties (Hooks et al. 2010) and its behavioral effects on H. glycines and M. incognita (Masler et al. 2017). We examined the host status of $T$. patula by inoculating it with $H$. glycines and $M$. incognita $\mathrm{J} 2$. Neither nematode reproduced on $T$. patula.

Marigold, pepper, and soybean seed were germinated in vermiculitefilled beakers in the greenhouse, and seedlings were maintained at $26^{\circ} \mathrm{C}$ with a photoperiod of 16 to $14 \mathrm{~h}$ of light and 8 to $10 \mathrm{~h}$ of darkness. Seedlings were harvested at 10 to 14 days (soybean) or 16 to 20 days (pepper and marigold) after germination. This harvesting approach provided root tips of similar size across the plant species. Roots were washed with tap water and rinsed with double-deionized water (DDW).

Collection of root tips. The terminal 5-mm tips from individual roots of $C$. annuum and $T$. patula root systems were collected. For G. max, which has a large taproot with lateral roots, the terminal $5 \mathrm{~mm}$ from individual lateral roots were collected. Freshly collected root tips were used for bioassay.

Preparation of root exudates. Seedlings were reared and harvested as described above. Root systems were washed with tap water, rinsed with DDW, and then incubated for $20 \mathrm{~h}(G$. $\max )$ or $1 \mathrm{~h}$ (C. annuum, G. max, and T. patula) at room temperature in $80 \mathrm{ml}$ of DDW. To compensate for differences in the sizes of root systems, incubation densities were typically approximately 0.5 plant system $\mathrm{ml}^{-1}$ of DDW for G. max and approximately 4 plant systems $\mathrm{ml}^{-1}$ for $C$. annuum and T. patula. Aliquots of the collected liquid (exudate) were dried under vacuum and stored at $-20^{\circ} \mathrm{C}$. Exudate doses are expressed in plant equivalent (plant eq) volume ${ }^{-1}$. Preliminary tests on exudate dose and nematode response yielded variable quantitative results. Qualitative results—attraction of $H$. glycines or repulsion of $M$. incognita - were consistent. Doses that yielded consistent quantitative results were used in all subsequent tests.

Preparation of root extracts. With marigold and pepper, entire rinsed root systems were cut into pieces and weighed fresh. For soybean, with its larger root system, only the lateral roots were collected and weighed. Root materials were extracted by hand in DDW using 1.5-ml polypropylene tubes (Axygen Inc., Union City, CA) fitted with polypropylene pestles. Extracts were centrifuged $(20 \mathrm{~min}$, $20,000 \times g, 5^{\circ} \mathrm{C}$ ) and supernatant volumes were measured. Because fresh root tissue yielded liquid during extraction, supernatant volume was greater than the volume of DDW used in extraction. For assays with fresh supernatant, concentrations are expressed as the original weight of fresh root material in total supernatant volume. A portion of fresh supernatant was used immediately in bioassays, with the remainder dried under vacuum and stored at $-20^{\circ} \mathrm{C}$. Extract doses in bioassays are expressed as milligrams of plant equivalent (mg eq) volume ${ }^{-1}$.

Evaluation of extracts of $G$. max lateral roots obtained from different root section positions. To examine for potential differences of $\mathbf{J} 2$ responses to materials obtained from locations along the length of the lateral root, soybean roots were collected, rinsed, and cut into several different section types using a sharp scalpel. Three terminal sections containing the root tip $(5,20$, and $40 \mathrm{~mm}$ from tip back toward the main root) and four nontip sections (5 to 10,10 to 15,15 to 20 , and 20 to $40 \mathrm{~mm}$ from the root tip) were prepared. Sections were counted and weighed, and section types pooled. The seven section pools were extracted in DDW and supernatants were collected as above. Supernatants were used fresh or dried and rehydrated as indicated for each experiment. Extract doses in bioassays are expressed as milligrams of tip section (mg eq) volume ${ }^{-1}$.

Reversed-phase fractionation. For broad fractionations, dried root exudates were rehydrated in $0.1 \%$ trifluoroacetic acid (TFA) and loaded (9 to 17 plant eq) onto $\mathrm{C}_{18}$ reversed-phase (RP) cartridge (SepPak; Millipore, Billerica, MA). Bound material was eluted in five steps with $1 \mathrm{ml}$ each of $0.1 \%$ TFA and an acetonitrile $\left(\mathrm{CH}_{3} \mathrm{CN}\right)$ series of $10,20,40$, and $80 \% \mathrm{CH}_{3} \mathrm{CN}$ in $0.1 \%$ TFA. All five fractions were dried under vacuum and rehydrated for bioassay as needed. For expanded separations, dried root exudates were rehydrated in $0.1 \%$
TFA and fractionated on an RP high-performance liquid chromatography (HPLC) system comprising an Agilent HP1200 liquid chromatograph (Agilent Technologies, Santa Clara, CA) fitted with an Agilent Eclipse $\mathrm{XDB} \mathrm{C}_{18}$ column (150 $\mathrm{mm}$ in length by $4.6 \mathrm{~mm}$ internal diameter). Mobile phase $\left(\mathrm{A}=0.1 \%\right.$ TFA and $\mathrm{B}=\mathrm{CH}_{3} \mathrm{CN}$ in $0.1 \%$ TFA) was run at $0.25 \mathrm{ml} \mathrm{min}^{-1}$, with $5 \% \mathrm{~B}$ from 0 to $5 \mathrm{~min}$ and 5 to $85 \%$ B from 5 to $45 \mathrm{~min}$. Typical sample input was 7.5 to 15 plant eq. Fractions $(500 \mu \mathrm{l})$ were collected at 2 -min intervals from 0 through $50 \mathrm{~min}$, and dried under vacuum. Dried fractions were rehydrated as needed for bioassay.

Bioassays. A solution of 23\% (wt/vol) Pluronic F-127 gel (SigmaAldrich, St. Louis) in DDW was prepared according to Wang et al. (2009b) and stored as liquid at $4^{\circ} \mathrm{C}$. To prepare nematodes for assay, infective $\mathrm{J} 2$ were suspended in DDW and mixed directly into the $\mathrm{F}-127$ solution at $10^{\circ} \mathrm{C}$ (necessary to maintain $\mathrm{F}-127$ as liquid). This mixture was dispensed into assay plate wells $(1 \mathrm{ml})$ or assay plates $(6 \mathrm{ml})$ such that $\mathbf{J} 2$ were homogeneously distributed. The solutions gelled for $10 \mathrm{~min}$ at room temperature, after which samples (root tips, sample dispensers) were added. All assays were conducted at $20^{\circ} \mathrm{C}$ in the dark,

Root tip bioassay. Attraction assays with root tips were according to Wang et al. (2009b). Briefly, $1 \mathrm{ml}$ of $23 \%$ F-127 containing $500 \mathrm{H}$. glycines $\mathrm{J} 2$ was loaded into wells of a 12 -well tissue culture plate (Corning Inc.), followed by the application of 1 C. annuum, G. $\max$, or T. patula root tip per well. All tips were $5 \mathrm{~mm}$ and plates were incubated in the dark at $20^{\circ} \mathrm{C}$. Nematodes touching each root tip were counted at 2 and $4 \mathrm{~h}$ after assay start, because nematodes were quickly attracted to the root tip. In total, 6 to 12 replicates were included in each experiment, and each experiment was repeated at least twice.

Root extract and exudate bioassays. Bioassays were modified from the methods described by (Wang et al. 2009a). F-127 solution (6 $\mathrm{ml}$ of 23\% gel) containing 1,800 freshly hatched $\mathrm{J} 2$ of $\mathrm{H}$. glycines or M. incognita were poured into 60-mm Petri plates (U.S.A. Scientific, Ocala, FL) at $10^{\circ} \mathrm{C}$. Root extracts or root exudates in DDW were mixed with F-127 to prepare sample solutions at a final gel concentration of $17.3 \%$. Control samples were prepared with DDW in F-127. Sample dispensers were modified 250- $\mu$ l pipette tips (wide-orifice; Mettler-Toledo Rainin, Oakland, CA), cut $2.3 \mathrm{~cm}$ from the small end to accommodate $100 \mu \mathrm{l}$ of sample. Two sample dispensers (replicates) were placed antiparallel and $1 \mathrm{~cm}$ apart in the center of the plate dispenser. Plates were incubated at $20^{\circ} \mathrm{C}$ for 20 $\mathrm{h}$, which allowed accumulation of $\mathrm{J} 2$ within the sample dispensers. $\mathrm{J} 2$ in dispensers were examined and counted using a Nikon SMZ1500 dissecting microscope with NIS-Elements imaging software (Nikon Instruments Inc., Melville, NY). Attraction or repulsion were quantified by calculating $\mathrm{J} 2$ in extract or exudate sample dispensers $(S)$ as a percentage of $\mathrm{J} 2$ in DDW control sample dispensers $(C)$ : $\left.\left(n \mathrm{~J} 2_{S}-n \mathrm{~J} 2_{C}\right) / n \mathrm{~J} 2_{C}\right) \times 100$. Two to three plates (four to six replicates) for each treatment were included in each experiment, and experiments were repeated at least twice.

Statistical analysis. JMP 12 software (SAS Institute Inc., Cary, NC) and GraphPad Prism 7 (GraphPad, LaJolla, CA) were used for the statistical analyses and data exploration. Individual means were compared using Student's two-tailed $t$ test and group means were compared by one-way analysis of variance (ANOVA) with Tukey's or Dunnett's multiple comparison tests as indicated.

\section{Results}

Response of $\mathbf{J} 2$ to root tips from three plant species. $M$. incognita and $H$. glycines $\mathrm{J} 2$ were differentially attracted to root tips prepared from C. annuum, G. max, and T. patula (Fig. 1). All three plants attracted $M$. incognita $\mathrm{J} 2$ into contact with root tips by $2 \mathrm{~h}$ after initiation of the in vitro assay, and no differences were observed among these plants in their levels of attraction to $M$. incognita (Fig. 1). In contrast, $H$. glycines $\mathrm{J} 2$ were attracted only to G. max. Attraction of $M$. incognita $\mathbf{J} 2$ to each of the three plant species increased with time. From 2 to $4 \mathrm{~h}$, percent increases were 68, 104, and $135(P<$ $0.05)$ for $C$. annuum, G. max, and T. patula, respectively. No changes in mean number of $H$. glycines $\mathrm{J} 2$ attracted to $G$. max were observed 


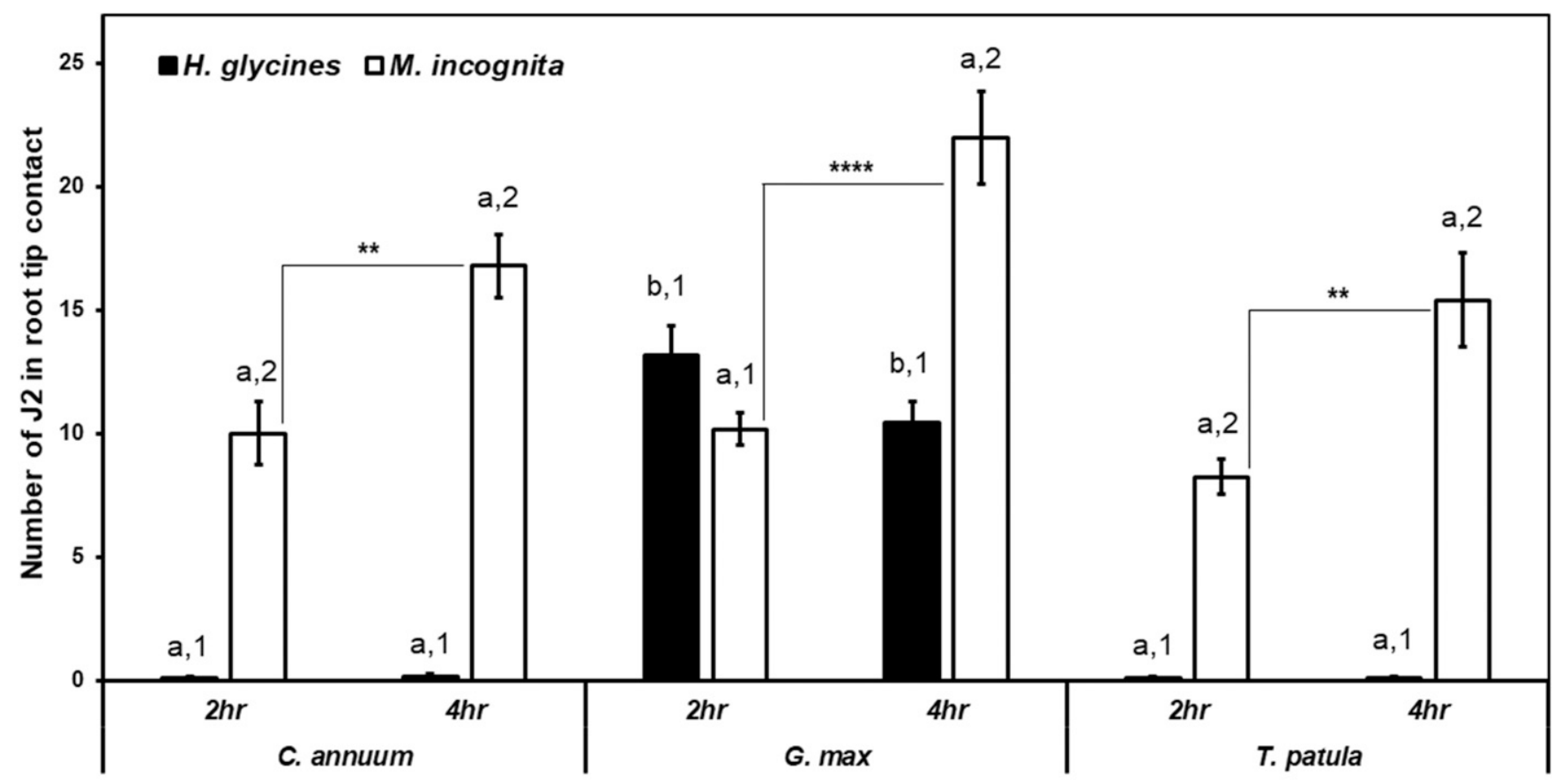

Root tip source

Fig. 1. Attraction of Heterodera glycines and Meloidogyne incognita infective juveniles $(\mathrm{J} 2)$ to root tips from three plant species in vitro. Root tips $(5 \mathrm{~mm})$ were prepared from freshly harvested roots and transferred to wells of a 12-well assay plate. Each well contained $1 \mathrm{ml}$ of Pluronic gel, approximately $500 \mathrm{~J} 2$, and 1 root tip. Plates were incubated at $20^{\circ} \mathrm{C}$ and $\mathrm{J} 2 \mathrm{in}$ contact with each tip were counted at 2 and $4 \mathrm{~h}$. Data are expressed as the mean number of $\mathrm{J} 2$ ( 5 to 10 replicates) touching root tips. For each nematode species, means within times and across plants were compared by one-way analysis of variance with Tukey's posttest. Means labeled with different letters are significantly different $(P<0.0001)$. For each species, means within plants and between times were compared using Student's $t$ test. Means connected by lines are significantly different at the levels indicated ${ }^{* *}$ and ${ }^{* * *}$ indicate $P<0.005$ and 0.0005 , respectively). Plant-time means were compared between species using Student's $t$ test. Means labeled with different numbers are significantly different $(P<0.0001)$.

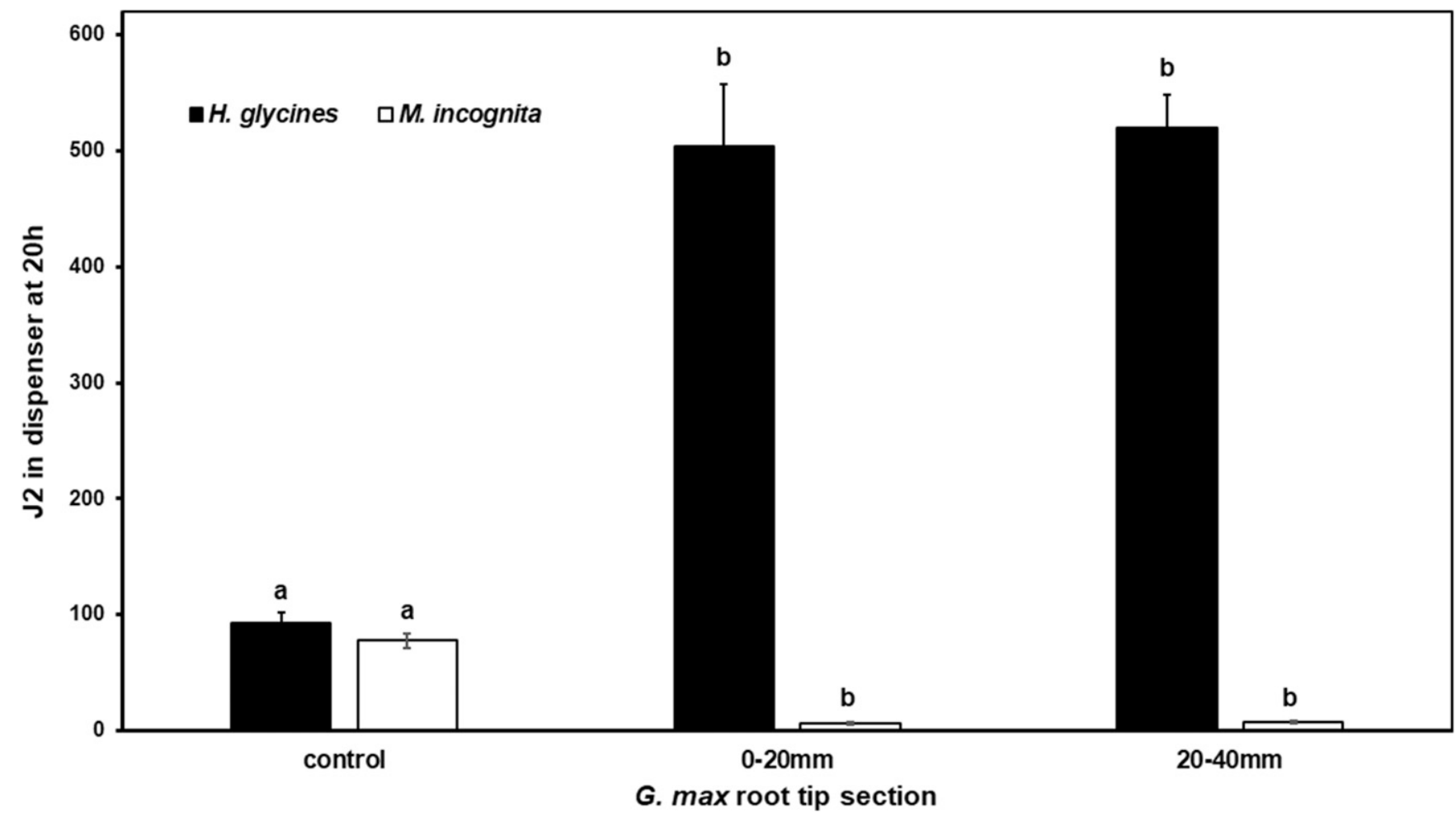

Fig. 2. Attraction of Heterodera glycines and Meloidogyne incognita infective juveniles (J2) to Glycine max lateral root extracts in vitro. Lateral root sections of 0 to 20 mm (includes root tip) and 20 to $40 \mathrm{~mm}$ were prepared as described and fresh extracts were used in the Pluronic gel-sample dispenser assay. Each assay plate contained $6 \mathrm{ml}$ of Pluronic gel, approximately $1,800 \mathrm{~J} 2$, and two sample dispensers. After $20 \mathrm{~h}$ at $20^{\circ} \mathrm{C}$, all J2 within the sample dispensers were counted. Means $(n=4$ to 10$)$ were compared using Student's $t$ test. Within nematode species and across treatments, means labeled with different letters are significantly different $(P<0.001)$. 
between 2 and $4 \mathrm{~h}$, and $H$. glycines remained unattracted to either T. patula or $C$. annuum.

Response of $\mathrm{H}$. glycines and $M$. incognita $\mathrm{J} 2$ to $G$. max root tip section extracts. Because $G$. $\max$ root tips were attractive to both $H$. glycines and $M$. incognita, we initiated further exploration by assessing the effects of $G$. max root content on $\mathrm{J} 2$ behavior, and included nontip sections of the lateral root. Fresh extract of 0-to-20$\mathrm{mm}$ sections of lateral roots ( 1 eq dispenser ${ }^{-1}$ ) was highly attractive to H. glycines $\mathrm{J} 2$, with a 5.5 -fold increase in $\mathrm{J} 2$ number over controls $(P<0.0001$; Fig. 2). In contrast, $M$. incognita $\mathrm{J} 2$ were 10 -fold less attracted than controls $(P<0.0001)$, suggesting a strong repellant effect. Results were the same with extract of sections (20 to $40 \mathrm{~mm}$ ) without the root tip (Fig. 2). The terminal 0-to-20-mm section of G. max lateral roots was examined further by comparing fresh and dried-rehydrated extracts. Attraction of $H$. glycines was the same for fresh $\left(504 \pm 53 \mathrm{~J} 2\right.$ dispenser $^{-1}$ at $\left.20 \mathrm{~h}\right)$ and rehydrated $(485 \pm$ 36) samples (Student's $t$ test, $n=4$ to $8, P>0.05$ ), suggesting that the attractants are not volatile. Dried-rehydrated extracts of the terminal 0 -to-20-mm section and four subsections ( 0 to 5 [tip section], 5 to 10,10 to 15 , and 15 to $20 \mathrm{~mm}$ ) were all equally attractive to $H$. glycines. Adjusting for fresh weights, means were $88 \pm 13,83 \pm 12,69 \pm$ $6,74 \pm 11$, and $72 \pm 11 \mathrm{~J} 2$ dispenser $^{-1} \mathrm{mg} \mathrm{eq}^{-1}$ respectively, for the five sections $(n=4$, one-way ANOVA, $P>0.05)$. In subsequent experiments, $G$. $\max$ root extracts were prepared using the 0 -to-20-mm section of lateral roots.

Response of $\mathbf{J} 2$ to root tip extracts from three plant species. Extracts of root systems from C. annuum and T. patula and 20-mm lateral root terminal sections from G. $\max$ were examined for attraction and

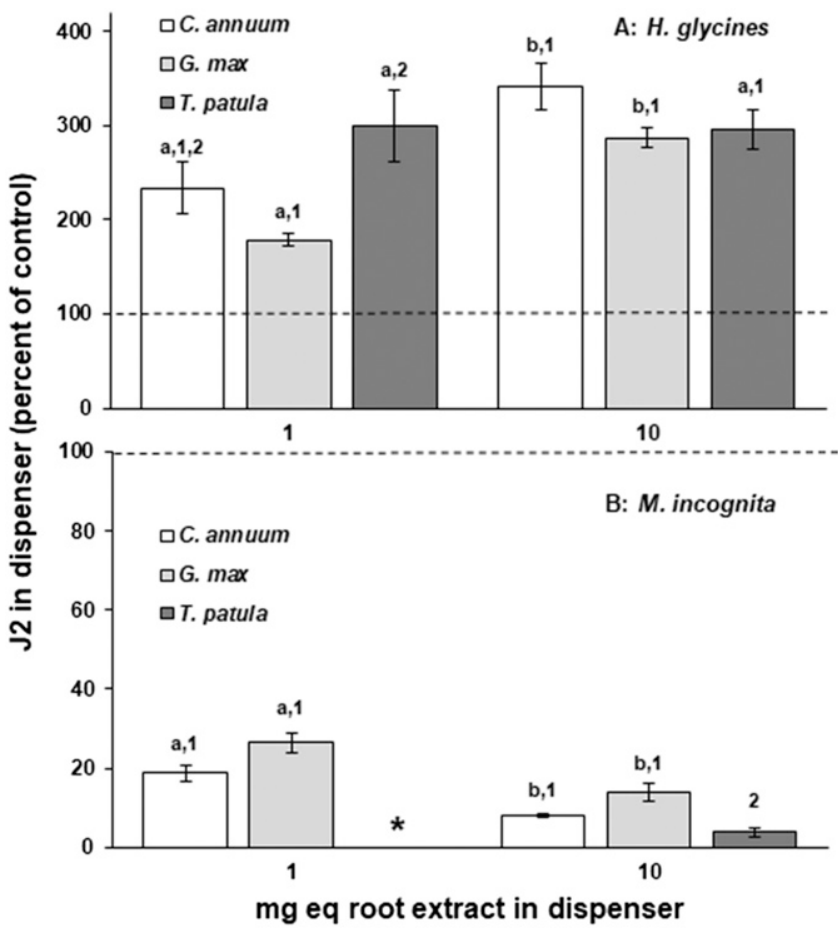

Fig. 3. Response of Heterodera glycines and Meloidogyne incognita infective juveniles (J2) to Capsicum annuum, Glycine max, and Tagetes patula root extracts tested at different doses in vitro. Rehydrated root extract supernatants were prepared in Pluronic gel and loaded into sample dispensers as described. Each assay plate contained $6 \mathrm{ml}$ of Pluronic gel, approximately 1,800 J2, and two sample dispensers. Doses tested were 1 or 10 root milligrams of plant equivalent (mg eq) dispenser ${ }^{-1}$, as indicated. After $20 \mathrm{~h}$ at $20^{\circ} \mathrm{C}$, all $\mathrm{J} 2$ within dispensers were counted. Data are expressed as the mean number of $\mathrm{J} 2$ in extract sample dispensers as a percentage of $\mathrm{J} 2$ in control sample dispensers. Means were compared using Student's $t$ test ( $n=4$ to 6 ; ${ }^{*}$ indicates that M. incognita-T. patula was not tested at 1 root $\mathrm{mg} \mathrm{eq}$ ). For each nematode, means within plants and between doses labeled with different letters are significantly different $(P<0.005)$. Means within doses and between plants that are labeled with different numbers are significantly different $(P<0.005)$. Horizontal dashed lines indicate response level equal to control. Values significantly greater than the control indicate attraction and those significantly less than the control indicate repulsion. repulsion effects on $H$. glycines and $M$. incognita $\mathrm{J} 2$ at two root $\mathrm{mg}$ equivalent levels (Fig. 3). All root extracts were attractive to H. glycines at both the 1 and $10 \mathrm{mg}$ eq doses $(P<0.005$; Fig. 3A). Attraction levels increased between $79 \%$ (1 mg eq G. $\max$ ) and $242 \%$ (10 $\mathrm{mg}$ eq $C$. annuum) relative to controls. In contrast, no extract was attractive to $M$. incognita at either dose (Fig. 3B). In fact, all $M$. incognita test means were significantly below control means $(P<0.005)$ by at least $70 \%$, indicating strong repellency. There were some dose effects with each nematode. For $H$. glycines, attraction increased 1.8-fold between 1 and $10 \mathrm{mg}$ eq with $C$. annuum and 2.4-fold with $G$. $\max$ $(P<0.05)$. No differences were observed between the $T$. patula doses. In $M$. incognita, apparent repulsion increased from 1 to $10 \mathrm{mg}$ eq by approximately 1.2 -fold $(P<0.05)$ with $C$. annuum and $G$. max.

Response of $\mathbf{J} 2$ to root exudates from three plant species. We examined the timing of exudate collection to determine whether reduced incubation times, which would more closely reflect root physiology postharvest than collecting for extended times, can be used in sample preparation. G. max root exudates collected at 1 and $20 \mathrm{~h}$ after root system soaking were tested for attractiveness to $H$. glycines (Table 1). There was no advantage for attracting $\mathrm{J} 2$ by incubating roots for $20 \mathrm{~h}$. In fact, more $\mathrm{J} 2$ were attracted to the 1-h exudate than

Table 1. Comparison of effects of Glycine max root exudate collection time on attraction of Heterodera glycines ${ }^{\mathrm{z}}$

\begin{tabular}{lcc}
\hline & \multicolumn{2}{c}{ Exudate collection } \\
\cline { 2 - 3 } Root equivalent dispenser $^{-1}$ & \multicolumn{1}{c}{$\mathbf{~ h}$} & $\mathbf{2 0 ~ h}$ \\
\hline Control & $80.8 \pm 5.0$ & $80.8 \pm 5.0$ \\
0.01 & $237.8 \pm 27.4 \mathrm{a} * *$ & $178.3 \pm 28.7 \mathrm{a} *$ \\
0.05 & $446.3 \pm 42.5 \mathrm{a} * * *$ & $335.8 \pm 39.5 \mathrm{a} * * *$ \\
0.1 & $601.5 \pm 24.4 \mathrm{a} * * * *$ & $450.8 \pm 46.8 \mathrm{~b} * * *$ \\
\hline
\end{tabular}

${ }^{\mathrm{z}} G$. $\max$ root exudates were collected at 1 and $20 \mathrm{~h}$ and processed as described. Dried and rehydrated samples were tested in the Pluronic gel plate assay for effects on $H$. glycines infective juvenile (J2) behavior. Assay plates were incubated $20 \mathrm{~h}$ at $20^{\circ} \mathrm{C}$ and all $\mathrm{J} 2$ within sample dispensers were counted. Data are expressed as mean J2 dispenser ${ }^{-1}(n=4)$. Means were compared using Student's $t$ test. At each dose and between collection times, means followed by different letters are significantly different $(P<0.05)$. Levels of significance of test means versus control mean are indicated $(*$, **, $* * *$, and $* * * *$ indicate $P<0.05,0.005,0.001$, and 0.0001 , respectively).

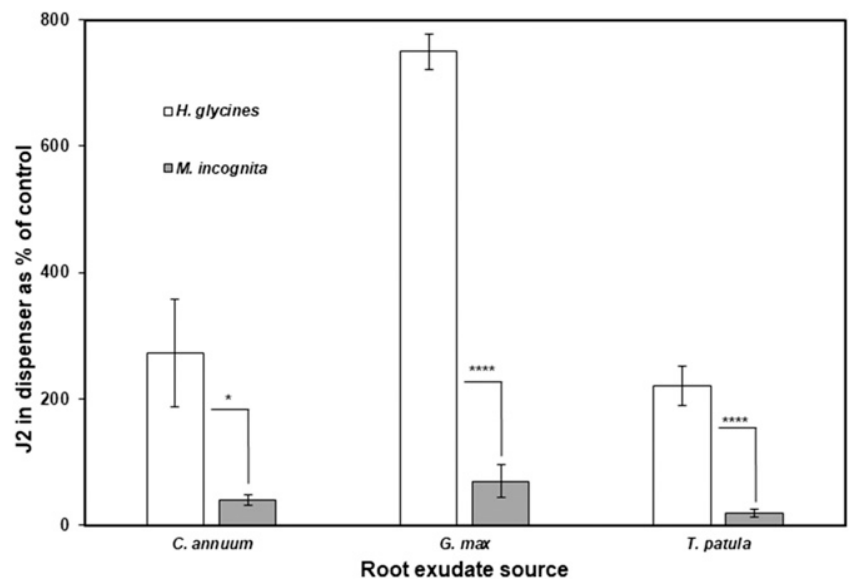

Fig. 4. Attraction of Heterodera glycines and Meloidogyne incognita infective juveniles (J2) to Capsicum annuum, Glycine max, and Tagetes patula root exudates in vitro. Fresh root systems were rinsed and incubated for $1 \mathrm{~h}$ at room temperature in double-deionized water, as described. Exudates were recovered, dried under vacuum, rehydrated in Pluronic gel-water, and loaded into sample dispensers as described. To adjust for differences in root masses, root exudate concentrations (plant equivalent dispenser ${ }^{-1}$ ) were 0.01 for $G$. max and 0.1 for $C$. annuum and $T$. patula. Assays were conducted in $60-\mathrm{mm}$ plastic dishes containing approximately $1,800 \mathrm{~J} 2$, as described. After $20 \mathrm{~h}$ at $20^{\circ} \mathrm{C}$, all $\mathrm{J} 2$ within dispensers were counted. Data are expressed as the mean number of $\mathrm{J} 2$ in dispensers as a percentage of water control. Means were compared using Student's $t$ test $(n=4$ to 6 ; for $M$. incognita-C. annuum, $n=2$ ). For each plant, levels of significant differences between nematodes are indicated ( ${ }^{*}$ and ${ }^{* \star * *}$ indicate $P<0.05$ and 0.001 , respectively). 
the 20 -h exudate at 0.1 plant eq $(P<0.05)$. Consequently, we used 1 -h exudates (which were within $2 \mathrm{~h}$ of harvest) for all subsequent assays. Exudates from C. annuum, G. max, and T. patula were highly attractive to $H$. glycines (Fig. 4). Percent increases in attraction over water controls $(P<0.05)$ were $156 \pm 21,627 \pm 25$, and $303 \pm 8$ for C. annuum, G. $\max$, and T. patula, respectively. All M. incognita responses were negative $(P<0.05)$, representing decreases of between 80 and $90 \%$ relative to water controls (data not shown).

Fractionation of root exudates. Stepwise $\mathrm{C}_{18}$ fractions of exudates from three plant species had similar but not identical attractive and repellent effects on $H$. glycines and $M$. incognita, respectively (Table 2). H. glycines was attracted $(P<0.005)$ to the most polar fraction (aqueous $0.1 \%$ TFA) from each of the plant species. In addition, equally strong attraction $(P<0.005)$ was also stimulated by the least polar fraction tested ( $80 \% \mathrm{CH}_{3} \mathrm{CN}$ in $0.1 \%$ TFA). Some of the intermediate polarity fractions from $C$. annuum and $T$. patula but not $G$. max were significantly $(P<0.05)$ attractive to $H$. glycines. $M$. incognita was strongly repelled $(P<0.0005)$ by each of the five fractions from $C$. annuum and $T$. patula but only the most polar fractions from G. max were strongly repellent to $M$. incognita. The 20 and $40 \%$ $\mathrm{CH}_{3} \mathrm{CN} G$. max fractions were moderately $(P<0.005)$ repellent whereas the $80 \% \mathrm{CH}_{3} \mathrm{CN}$ fraction had no effect.

The clear behavioral differences of $H$. glycines and $M$. incognita $\mathrm{J} 2$ that were observed with whole exudates were retained by fractionated exudates exposed to low $\mathrm{pH}$ and organic solvent. These characteristics were examined further using RP-HPLC with gradient separation. The strong attractive $(H$. glycines) and repellent $(M$. incognita) activities detected in the stepwise separations are present in the column fractions (Fig. 5). In fact, attractive and repellent materials are present in the same two polar fractions (numbers 4 and 5), eluting between 9 and $15 \% \mathrm{CH}_{3} \mathrm{CN}$, for all three plants. This is consistent with results from the cartridge tests. It is curious that the relatively weaker $H$. glycines attractive effect of the G. max TFA cartridge fraction (versus C. annuum and T. patula; Table 2) is reflected in the column fractions. Fractions 4 and 5 from $C$. annuum and T. patula (Fig. 5A and E) are attractive whereas only G. max fraction 4 is attractive (Fig. 5C). Quite unexpected was the absence of any attractive activity in fractions eluting above $15 \% \mathrm{CH}_{3} \mathrm{CN}$ from all three plants. This is in stark contrast with the stepwise results (Table 2). A similar condition was observed with $M$. incognita repellency by C. annuum (Fig. 5B), where only the polar fractions (numbers 4 and 5) were repellent. In contrast, G. $\max$ and T. patula contain repellent materials eluting in both polar and less polar fractions (Fig. 5D and F), with fractions 12 and 13 (41 to $47 \% \mathrm{CH}_{3} \mathrm{CN}$ ) showing consistent effects.

\section{Discussion}

$H$. glycines and $M$. incognita infective $\mathrm{J} 2$ exhibit varying behaviors when exposed to plant root signals presented in a variety of forms and derived from different plant species. These different behaviors expose fundamental chemoreceptive differences between $H$. glycines and $M$. incognita. The large array of chemicals present in and released from roots contain both attractants and repellants to plant-parasitic nematodes (Reynolds et al. 2011; Wuyts et al. 2006; Xu et al. 2015), and the chemical signals expressed can vary not only between plant species but also between same-species cultivars (Yang et al. 2016; Zhao et al. 2000). Our observations with whole root tips presumably illustrate differences in both root phytochemicals and perception by $H$. glycines and $M$. incognita. Because the root tips in our assays were cut from root systems, they represent mechanically disrupted, wounded tissue that may be expected to present different chemical cocktails than those released by intact systems. For example, activation of the ethylene pathway through wounding can invoke varied attraction or repulsion responses by Heterodera and Meloidogyne spp. (Fudali et al. 2013; Hu et al. 2017). Nevertheless, $H$. glycines and $M$. incognita, with relatively narrow and wide host preferences, respectively (Lilley et al. 2005), behaved rather predictably in our assays with regard to attraction.

The aggressive disruption of root tissue through mechanical extraction should produce samples containing secondary metabolite mixtures to which $\mathrm{J} 2$ are not normally exposed. Release of alkaloids, phenolics, proteins, terpenoids and many other phytochemicals can be expected (Bacetty et al. 2009; Chitwood 2002; De-la-Peña et al. 2012; Pegard et al. 2005; Reynolds et al. 2011; Wuyts et al. 2006; Yang et al. 2016). In addition, enzymes such as those which hydrolyze glucosinolates into active products (Gimsing and Kirkegaard 2009) may be activated, adding to the complexity. In this chemical context, it is notable that the two nematodes behave completely differently. The repulsion of $M$. incognita $\mathrm{J} 2$ is in complete opposition to their attraction to intact root tips, whereas the attraction of $\mathrm{H}$. glycines $\mathrm{J} 2$ to root extracts is an extension of their root tip behaviors. Without chemical characterization of the extracts, and with bioassay limitations, specific conclusions regarding the molecules involved in the $\mathrm{J} 2$ behaviors cannot be drawn. However, because the results are consistent across three plant species and 10-fold different concentrations, they do, in fact, suggest strikingly different chemoreceptive systems in the two nematodes. Root extract slurries, which contained all materials from disrupted roots (Masler et al. 2017), elicited behaviors from $H$. glycines and $M$. incognita similar to those reported here with extract supernatants rather than to root tips. This suggests that root tissue disruption itself causes the changes in behavior relative to root tips.

Manipulations of root tips illustrate complexities of phytochemical source and nematode behavior. $H$. glycines $\mathbf{J} 2$ are strongly attracted to the apical root tip of G. max seedlings (Hu et al. 2017) and to cut apical root tips (this work). It was somewhat surprising, then, that extracts from different locations along the lateral roots of $G$. max were

Table 2. High- and low-polarity active components in root exudates of three plant species as detected with Heterodera glycines and Meloidogyne incognita attraction and repellency bioassays ${ }^{\mathrm{z}}$

\begin{tabular}{|c|c|c|c|c|c|c|}
\hline \multirow[b]{3}{*}{ Nematode, plant } & \multicolumn{6}{|c|}{ Number of nematode $\mathrm{J} 2$ per dispenser } \\
\hline & \multirow[b]{2}{*}{ Control } & \multirow[b]{2}{*}{ TFA } & \multicolumn{4}{|c|}{ Cartridge fraction } \\
\hline & & & 10 & 20 & 40 & 80 \\
\hline \multicolumn{7}{|l|}{ H. glycines } \\
\hline Capsicum аппиит & 135 & $477 * * * *$ & 215 & $348 * *$ & $352 * *$ & $517 * * * *$ \\
\hline Glycine $\max$ & 190 & $751 * *$ & 278 & 224 & 487 & $618^{* *}$ \\
\hline Tagetes patula & 117 & $409 * * * *$ & $295 * * * *$ & $181^{*}$ & $257 * * *$ & $485^{* * * *}$ \\
\hline \multicolumn{7}{|l|}{ M. incognita } \\
\hline C. аппиит & 82 & $4 * * * *$ & $25 * * * *$ & $42 * * * *$ & $25 * * * *$ & $6^{* * * *}$ \\
\hline G. $\max$ & 188 & $16^{* * *}$ & $36 * * *$ & $84 * *$ & $111^{*}$ & 129 \\
\hline T. patula & 82 & $3 * * * *$ & $7 * * * *$ & $16^{* * *}$ & $5 * * * *$ & $8 * * * *$ \\
\hline
\end{tabular}

${ }^{\mathrm{z}}$ Root exudates were applied to $\mathrm{a}_{18}$ SepPak cartridge system and eluted stepwise with $0.1 \%$ trifluoroacetic acid (TFA) and 10, 20, 40, and $80 \%$ concentrations of acetonitrile in $0.1 \%$ TFA, as described. Dried and rehydrated fractions were tested in the Pluronic gel plate assay for effects on infective juvenile (J2) behavior. Assay doses were 0.25 plant equivalent dispenser ${ }^{-1}$ for $C$. annuum, 0.1 for $G$. max , and 0.45 for T. patula. Assay plates were incubated $20 \mathrm{~h}$ at $20^{\circ} \mathrm{C}$, and all $\mathrm{J} 2$ within sample dispensers were counted. Data are expressed as mean $\mathrm{J}_{2}$ dispenser $^{-1}(n=2$ to 4$)$. For each nematode-plant group, test means were compared against controls by one-way analysis of variance with Dunnett's multiple comparison. Levels of significant differences from control are indicated (*,**, $* * *$, and $* * * *$ indicate $P<0.05,0.005$, and 0.0005 and $P=0.0001$, respectively). 
equally attractive to $H$. glycines $\mathrm{J} 2$. Because extracts are the result of aggressive and disruptive methods, and root exudates can be regarded as the products of a more passive and realistic approach, an argument can be made that exudates, although still artificial substitutes for intact roots, more closely reflect what nematodes may be exposed to in the rhizosphere compared with extracts. It is notable that, in examining the effects of potato root diffusate (i.e., exudate) on Globodera rostochiensis hatch, Rawsthorne and Brodie (1986) found that, although diffusate was produced primarily by cells at the root tip, active diffusate was nevertheless produced along the entire length of the root. It was revealing, then, that $\mathrm{J} 2$ behavior in the presence of exudates in our experiments mimicked the responses to extracts rather than to root tips. Zhao et al. (2000) demonstrated that
M. incognita $\mathrm{J} 2$ were attracted to root tips from pea (Pisum sativum) and alfalfa (Medicago sativa 'Thor') but were repelled by root exudates from these same plants. They identified root tip border cells as the source of attractants, because removal of the cells by washing removed the attraction. Because loss of attractant cannot explain repellency as opposed to simple nonattraction, they cite the presence of positive and negative effectors in root exudates that may affect behavior. Such materials freely accessible in an exudate, as in our experiments, must present a different challenge to the nematode than the root tip.

Yang et al. (2016) demonstrated that the responses of Meloidogyne incognita to exudates of three cultivars of tomato were correlated with the susceptibility of the cultivar to $M$. incognita. Exudate of a
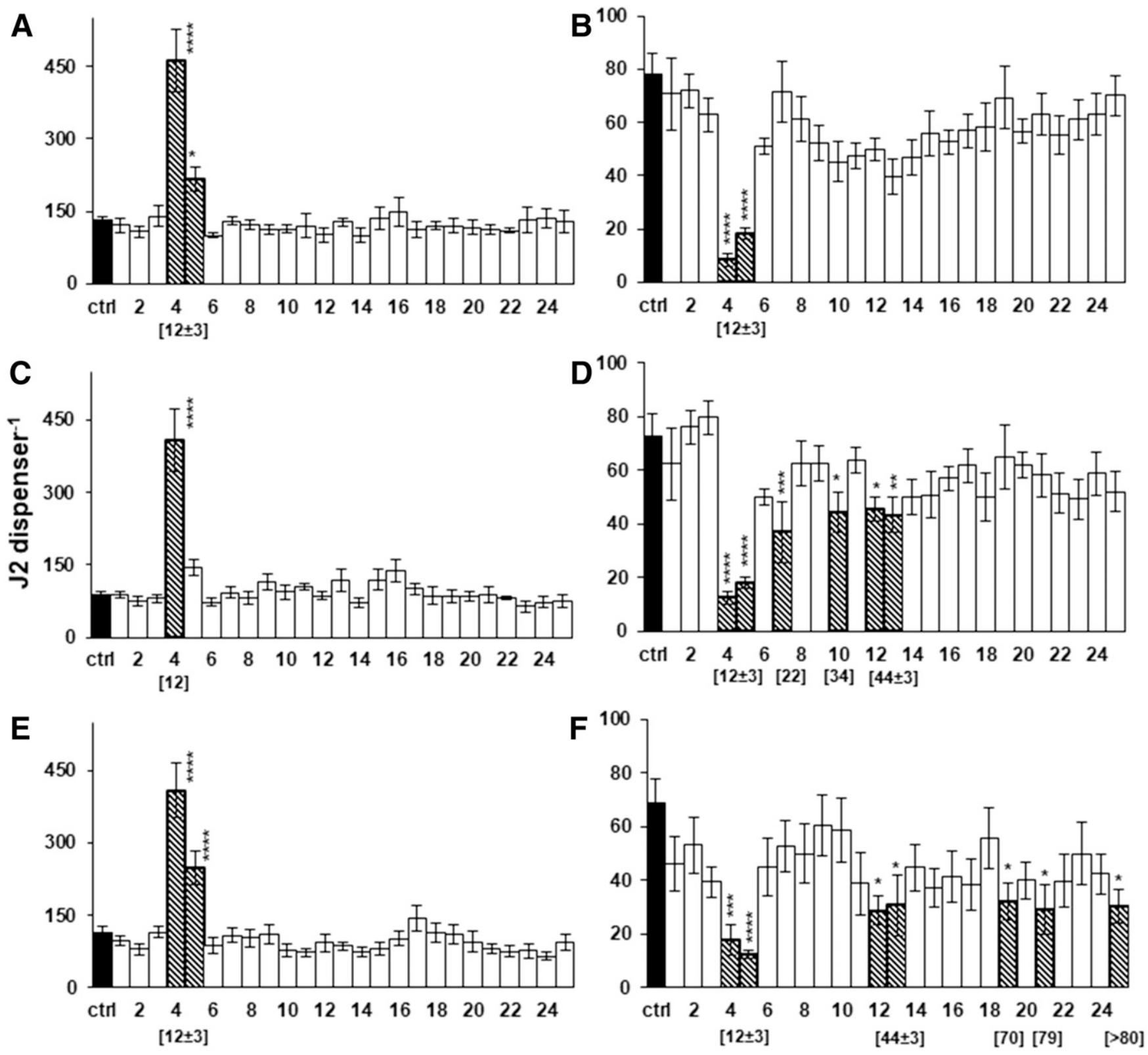

Fraction Number

Fig. 5. Fractionation of attractant and repellent materials from root exudates of Capsicum annuum, Glycine max, and Tagetes patula by reversed-phase (RP) high-performance liquid chromatography. Exudates were prepared as described, dried, rehydrated in $0.1 \%$ trifluoroacetic acid, and injected onto a $\mathrm{C}_{18} \mathrm{RP}$ column. Fractions were collected, dried, and dissolved in Pluronic gel-water mixtures for bioassay in 60 -mm plastic dishes containing approximately 1,800 infective juveniles (J2), as described. Concentrations of plant equivalent dispenser ${ }^{-1}$ were 0.13 to 0.25 . Data are expressed as the mean number of $\mathrm{J} 2$ present in sample dispensers after $20 \mathrm{~h}$ at $20^{\circ} \mathrm{C}$ for each of 25 fractions (open bars) plus controls (ctrl; black bars). Fractions with significant attraction or repulsion versus control are indicated by shaded bars. Data are summarized across four independent experiments for each nematode species-plant combination ( $n=6$ to 8 of each fraction). Fraction means are compared with control means using one-way analysis of variance with Dunnett's. Levels of significance from control are indicated $\left(^{*},{ }^{* *},{ }^{* * *}\right.$, and ${ }^{* * * *}$ indicate $P<0.05,0.01,0.001$, and 0.001 , respectively). Numbers in parentheses below x-axes indicate gradient position $\left(\% \mathrm{CH}_{3} \mathrm{CN}\right.$ range) of active fractions. The six profiles indicate Heterodera glycines $\times \mathbf{A}, \mathrm{C}$. annuum; $\mathbf{C}, \mathrm{G}$. max; and $\mathbf{E}$, T. patula; and Meloidogyne incognita $\times$ B, C. annuum; D, G. max; and F, T. patula. 
highly resistant cultivar repelled $M$. incognita in vitro whereas exudate of a susceptible cultivar was highly attractive. Exudate of a third cultivar of moderate resistance had a neutral effect, neither attracting nor repelling. The authors suggest that differences in root exudate composition can be an important contributing factor to resistance. The sensitivity of $\mathbf{J} 2$ to positive and negative signals was illustrated by Reynolds et al. (2011) using intact seedlings of tomato (Solanum lycopersicum) and rice (Oryza sativa) as exudate sources in a chemotaxis assay system designed to measure $\mathrm{J} 2$ directional efficiency in host seeking. Although M. incognita and M. graminicola were attracted to each plant, the $\mathrm{J} 2$ proceeded to their preferred host (M. incognita to tomato and $M$. graminicola to rice) by the shortest possible path whereas longer paths were taken to the less-preferred host. The authors suggest that the $\mathrm{J} 2$ negotiated complex mixtures of repellents and attractants in selecting the best paths to the host. In addition, they hypothesized that nematodes with relatively broad host ranges (e.g., M. incognita) depend upon general plant cues, consisting of appropriate blends and concentrations of semiochemicals, for host location. In contrast, they suggest that nematodes with narrower host ranges (e.g., M. graminicola and cyst nematodes) are more specific in their responses to chemical signals.

Exudates from C. annuum, G. max, and T. patula contain attractant and repellent compounds that are water soluble, nonvolatile, and stable at low $\mathrm{pH}$. Stepwise chromatographic separations clearly indicate the presence of highly polar compounds and of compounds of decreasing relative polarities, all of which have effects on behavior. These highly polar compounds consistently elicit strong attraction in $H$. glycines and repellency by $M$. incognita, which is consistent with reports by Papademetriou and Bone (1983) and Diez and Dusenbery (1989) with polar materials eluted from $\mathrm{C}_{18}$ substrates. Papademetriou and Bone (1983) reported that H. glycines were attracted to a G. max fraction whereas Diez and Dusenbery (1989) reported repellency of $M$. incognita by tomato exudate eluent. The least polar $(80 \%)$ fractions we collected from each of the three plant species retained the $H$. glycines attractive and $M$. incognita repellent properties, except for the G. max noneffect on $M$. incognita. More extensive separations of the $80 \%$ fractions by RP-HPLC revealed that much polar material remained and that the early eluting column fractions retained the attraction or repulsion properties of the input samples. It is probable that the early eluting fractions comprised complex mixtures of active attractants and repellants. Additional methods such as those used by Devine and Jones (2003) with potato root leachate are needed for further fractionation and characterization.

Results with fractions eluting at higher $\mathrm{CH}_{3} \mathrm{CN}$ concentrations are noteworthy. The complete loss of $H$. glycines attraction may indicate quantitative or qualitative deficiencies. For example, distribution of attractant between neighboring fractions may reduce detectable amounts in each fraction below a necessary minimum. Another possibility is that fractionation separates active compounds that usually act synergistically to attract. Preliminary attempts at fraction mixing (data not shown) have had inconsistent results. Nevertheless, the different chemical mixtures between the $80 \%$ input and the subsequent column fractions significantly affected $H$. glycines behavior. This may reflect specific semiochemical mixture requirements by $H$. glycines, similar to the concept suggested by Reynolds et al. (2011). In contrast to $H$. glycines attraction, $M$. incognita repulsion by lower polarity fractions from G. max and T. patula presents more potential for compound identification, especially because the same two fractions from each species are consistently repellent. The results with C. annuum might be explained using the same argument given for the absence of $H$. glycines attraction. It is particularly interesting that the G. max fractions are repellent given that the $80 \%$ source sample was not. In a variant of the argument of loss of attractiveness with $H$. glycines, fractionation may reduce conflicting signals and allow detection of repellent. Detection of repellency in $G$. $\max$ and $T$. patula column fractions is consistent with small lipophilic molecules prepared by solid-phase and solvent extractions of $S$. lycopersicum and $O$. sativa exudates that affect Meloidogyne spp. mobility, with possible repellent effects (Dutta et al. 2012).
A major advance in the discovery of natural control compounds will come from understanding the behaviors of plant-parasitic nematodes in the rhizosphere. This chemically complex environment for signal exchange (Baetz and Martinoia 2014; Bais et al. 2006; Chaparro et al. 2013) presents both challenge and reward for compound discovery. Bioassay development incorporating comparative approaches, including varied plant and nematode species and selected plant metabolic ages, will contribute to this discovery.

\section{Literature Cited}

Bacetty, A. A., Snook, M. F., Glenn, A. E., Noe, J. P., Nagabhyru, P., and Bacon, C. W. 2009. Chemotaxis disruption in Pratylenchus scribneri by tall fescue root extracts and alkaloids. J. Chem. Ecol. 35:844-850.

Baetz, U., and Martinoia, E. 2014. Root exudates: The hidden part of plant defense. Trends Plant Sci. 19:90-98.

Bais, H. P., Weir, T. L., Perry, L. G., Gilroy, S., and Vivanco, J. M. 2006. The role of root exudates in rhizosphere interactions with plants and other organisms. Annu. Rev. Plant Biol. 57:233-266.

Chaparro, J. M., Badri, D. V., Bakker, M. G., Sugiyama, A., Manter, D. K., and Vivanco, J. M. 2013. Root exudation of phytochemicals in Arabidopsis follows specific patterns that are developmentally programmed and correlate with soil microbial functions. PLoS One 8:e55731.

Chitwood, D. J. 2002. Phytochemical based strategies for nematode control. Annu. Rev. Phytopathol. 40:221-249.

Dalzell, J. J., Kerr, R., Corbett, M. D., Fleming, C. C., and Maule, A. G. 2011. Novel bioassays to examine the host-finding ability of plant-parasitic nematodes. Nematology 13:211-220.

De-la-Peña, C., Badri, D. V., and Loyola-Vargas, V. M. 2012. Plant root secretions and their interactions with neighbors. Pages 1-26 in: Secretions and Exudates in Biological Systems. Signaling and Communication in Plants, Vol. 12. J. M. Vivanco and F. Baluška, eds. Springer-Verlag, Berlin, Heidelberg.

Devine, K. J., and Jones, P. W. 2003. Investigations into the chemoattraction of the potato cyst nematodes Globodera rostochiensis and G. pallida towards fractionated potato root leachate. Nematology 5:65-75.

Diez, J. A., and Dusenbery, D. B. 1989. Repellent of root-knot nematodes from exudate of host roots. J. Chem. Ecol. 15:2445-2455.

Dutta, T. K., Powers, S. J., Gaur, H. S., Birkett, M., and Curtis, R. H. C. 2012. Effect of small lipophilic molecules in tomato and rice root exudates on the behavior of Meloidogyne incognita and M. graminicola. Nematology 14:309-320.

Farnier, K., Bengtsson, M., Becher, P. G., Witzell, J., Witzgall, P., and Manduric, S. 2012. Novel bioassay demonstrates attraction of the white potato cyst nematode Globoders pallida (Stone) to non-volatile and volatile host plant cues. J. Chem. Ecol. 38:795-801.

Fudali, S. L., Wang, C., and Williamson, V. M. 2013. Ethylene signaling pathway modulates attractiveness of host roots to the root-knot nematode Meloidogyne hapla. Mol. Plant-Microbe Interact. 26:75-86.

Gimsing, A. L., and Kirkegaard, J. A. 2009. Glucosinolates and biofumigation: Fate of glucosinolates and their hydrolysis products in soil. Phytochem. Rev. 8:299-310.

Grundler, F., Schnibbe, L., and Wyss, U. 1991. In vitro studies on the behavior of second-stage juveniles of Heterodera schachtii (Nematoda: Heteroderidae) in response to host plant root exudates. Parasitology 103:149-155.

Hooks, C. R. R., Wang, K.-H., Ploeg, A., and McSorley, R. 2010. Using marigold (Tagetes spp.) as a cover crop to protect crops from plant-parasitic nematodes. Appl. Soil Ecol. 46:307-320.

Hu, Y., You, J., Li, C., Williamson, V. M., and Wang, C. 2017. Ethylene response pathway modulates attractiveness of plant roots to soybean cyst nematode Heterodera glycines. Sci. Rep. 7: Article 41282.

Jones, J. 2002. Nematode sense organs. Pages 353-368 in: The Biology of Nematodes. D. L. Lee, ed. Taylor \& Francis, London.

Jones, J. T., Haegeman, A., Danchin, E. G. J., Gaur, H., Helder, J., Jones, M. G. K., Kikuchi, T., Manzanilla-Lopez, R., Palomares-Rius, J. E., Wesemael, W. M. L., and Perry, R. N. 2013. Top 10 plant-parasitic nematodes in molecular plant pathology. Mol. Plant Pathol. 14:946-961.

Lilley, C. J., Atkinson, H. J., and Urwin, P. E. 2005. Molecular aspects of cyst nematodes. Mol. Plant Pathol. 6:577-588.

Masler, E. P., Rogers, S. T., and Hooks, C. R. R. 2017. Behavioral differences of Heterodera glycines and Meloidogyne incognita infective juveniles exposed to root extracts in vitro. Nematology 19:175-183.

Nicol, J. M., Turner, S. J., Coyne, D. L., den Nijs, L., Hockland, S., and Tanha Maafi, Z. 2011. Current nematode threats to world agriculture. Pages 21-43 in: Genomics and Molecular Genetics of Plant-Nematode Interactions. J. Jones, G. Gheysen, and C. Fenoll, eds. Springer, Dordrecht, The Netherlands.

Ntalli, N., and Caboni, P. 2012. Botanical nematicides: A review. J. Agric. Food Chem. 60:9929-9940.

Papademetriou, M. K., and Bone, L. W. 1983. Chemotaxis of larval soybean cyst nematode, Heterodera glycines Race 3, to root leachates and ions. J. Chem. Ecol. 9:387-396.

Pegard, A. P., Brizzard, G., Fazari, A., Soucaze, O., Abad, P., and DjianCaporalino, C. 2005. Histological characterization of resistance to different root-knot nematode species related to phenolics accumulation in Capsicum annuum. Phytopathology 95:158-165. 
Perry, R. N. 1996. Chemoreception in plant parasitic nematodes. Annu. Rev. Phytopathol. 34:181-199.

Perry, R. N. 1997. Plant signals in nematode hatching and attraction. Pages 38-50 in: Cellular and Molecular Aspects of Plant-Nematode Interactions. C. Fenoll, F. M. W. Grundler, and S. A. Ohl, eds. Kluwer Academic Publishers, Dordrecht, The Netherlands.

Perry, R. N., and Curtis, R. H. C. 2013. Behaviour and sensory perception. Pages 246-273 in: Plant Nematology, 2nd ed. R. N. Perry and M. Moens, eds. CAB International, Wallingford, Oxfordshire, UK.

Rasmann, S., Ali, J. G., Helder, J., and van der Putten, W. H. 2012. Ecology and evolution of soil nematode chemotaxis. J. Chem. Ecol. 38:615-628.

Rawsthorne, D., and Brodie, B. B. 1986. Relationship between root growth of potato, root diffusate production, and hatching of Globodera rostochiensis. J. Nematol. 18:379-384.

Reynolds, A. M., Dutta, T. K., Curtis, R. H. C., Powers, S. J., Gaur, H. S., and Kerry, B. R. 2011. Chemotaxis can take plant-parasitic nematodes to the source of a chemo-attractant via the shortest possible routes. J. R. Soc. Interface 8:568-577.

Sardanelli, S., and Kenworthy, W. J. 1997. Soil moisture control and direct seeding for bioassay of Heterodera glycines on soybean. Suppl. J. Nematol. 29:625-634.

Wang, C., Bruening, G., and Williamson, V. M. 2009a. Determination of preferred $\mathrm{pH}$ for root-knot nematode aggregation using Pluronic F-127 gel. J. Chem. Ecol. 35:1242-1251.
Wang, C., Lower, S., Thomas, V. P., and Williamson, V. M. 2010. Root-knot nematodes exhibit strain-specific clumping behavior that is inherited as a simple genetic trait. PLoS One 5:e15148.

Wang, C., Lower, S., and Williamson, V. M. 2009b. Application of Pluronic gel to the study of root-knot nematode behaviour. Nematology 11:453-464.

Winter, M. D., McPherson, M. J., and Atkinson, H. J. 2002. Neuronal uptake of pesticides disrupts chemosensory cells of nematodes. Parasitology 125: 561-565.

Wuyts, N., Swennen, R., and De Waele, D. 2006. Effects of plant phenylpropanoid pathway products and selected terpenoids and alkaloids on the behaviour of the plant-parasitic nematodes Radopholus similis, Pratylenchus penetrans and Meloidogyne incognita. Nematology 8:89-101.

Xu, Z., Zhao, Y.-Q., Yang, D.-J., Sun, H.-J., Zhang, C.-L., and Xie, Y.-P. 2015 Attractant and repellent effects of sweet potato root exudates on the potato rot nematode, Ditylenchus destructor. Nematology 17:117-124.

Yang, G., Zhou, B., Zhang, X., Zhang, Z., Wu, Y., Zhang, Y., Lu, S., Zou, Q., Gao, Y., and Teng, L. 2016. Effects of tomato root exudates on Meloidogyne incognita. PLoS One 11:e0154675.

Zhao, X., Schmitt, M., and Hawes, M. C. 2000. Species-dependent effects of border cell and root tip exudates on nematode behavior. Phytopathology 90: $1239-1245$ 